\title{
Comportamientos ambientales en el sector turismo: revisión de tema
}

Environmental behaviors in the tourism sector: topic review

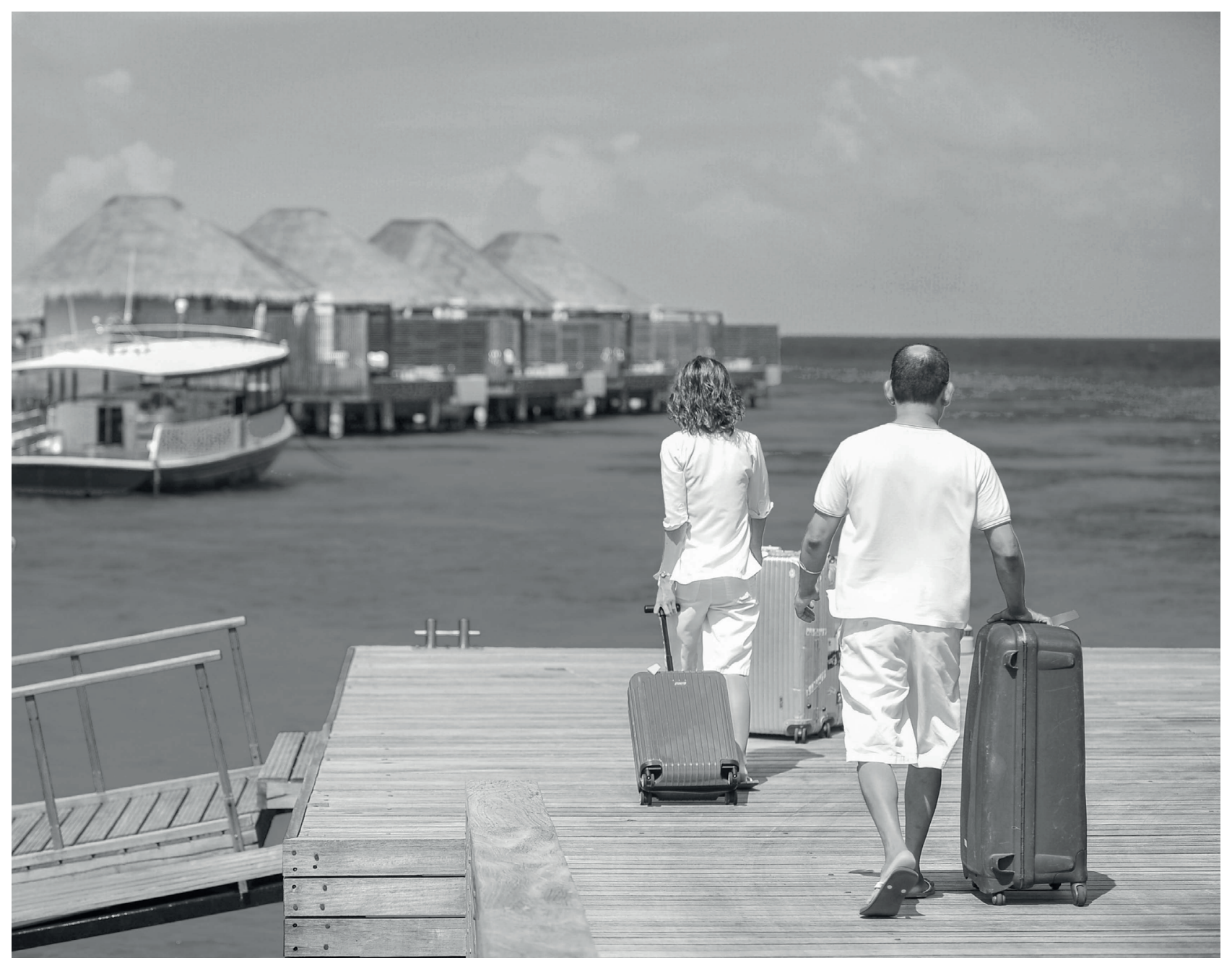




\title{
Comportamientos ambientales en el sector turismo: revisión de tema ${ }^{1}$
}

\author{
Environmental behaviors in the tourism sector: topic review
}

\author{
Cindy Tatiana Daza², Sonia Astrid Reyes Duarte ${ }^{3}$ \\ Artículo recibido en abril 27 de 2020; artículo aceptado en julio 03 de 2020
}

\begin{abstract}
Este artículo puede compartirse bajo la Licencia Creative Commons Atribución-NoComercial-Compartirlgual 4.0 Internacional y se referencia usando el siguiente formato: Daza, C. T. y Reyes, S. A. (2021). Comportamientos ambientales en el sector turismo: revisión de tema. I+D Revista de Investigaciones, 16(2), 8-17.https://doi.org/10.33304/revinv.v16n22021001.
\end{abstract}

\section{Resumen}

Se realizó una revisión sobre prácticas ambientales en el sector turismo, con el fin de generar conocimiento que contribuya a mejorar el comportamiento ambiental del sector. Para esto se utilizó una ecuación de búsqueda, en español, en la base de datos Scielo, y en inglés, en la base de datos Scopus. Se incluyeron artículos publicados durante los últimos 5 años. El resultado final fueron 30 artículos que cumplían los criterios de inclusión para este estudio. El enfoque del artículo es novedoso porque aborda de forma integral, según la revisión bibliográfica, los diferentes modelos que buscan predecir el comportamiento ambiental, lo cual permite tener las bases teóricas para formular estrategias más completas enfocadas al cambio de comportamiento. Se concluye que es necesario utilizar un abordaje integral en las estrategias que buscan la promoción de comportamientos ambientales en el sector turismo.

Palabras clave: Comportamientos, conservación ambiental, ecología, medio ambiente, turismo.

\section{Abstract}

An environmental behavior review was done in order to generate knowledge that contributes to improve the environmental behaviors in the tourism sector. For this reason, a search equation was used in Spanish, the database Scielo, and in English, the database Scopus. 30 articles meeting the inclusion criteria for this study were the result of the search mentioned, where articles published during the past 5 years were included. Hence that the approach of the article is innovative because, according to the bibliographic review, it comprehensively addresses the different models that seek to predict environmental behavior which allows to have the theoretical bases to formulate more

\footnotetext{
${ }^{1}$ Artículo de revisión, enfoque cualitativo, resultado de un trabajo de investigación en curso, área de Ciencias sociales y del comportamiento, subárea gestión ambiental empresarial, desarrollado en el grupo de investigación SINERGIA, fue financiado por la Corporación Universidad de Investigación y Desarrollo (UDI). Dirección: Calle 9 n. 23-55 (Bucaramanga, Colombia). PBX: (60) (7) 6352525. Fecha de inicio: Febrero de 2020.

${ }^{2}$ Magíster en Desarrollo Sostenible y Medio Ambiente de la Universidad de Manizales. Docente investigadora del Grupo de Investigación SINERGIA Universidad de Investigación y Desarrollo -UDI (Bucaramanga, Colombia). Dirección: Calle 9 n. ${ }^{\circ}$ 23-55 PBX: (60) (7) 6352525. ORCID ID: https://orcid. org/0000-0003-2749-3602.Correo electrónico institucional: cdaza1@udi.edu.co // cindy.daza.r@gmail.com.

${ }^{3}$ Magíster en Sistemas Integrados de Gestión de la Universidad Internacional de la Rioja. Docente investigadora del Grupo de Investigación SARET, Universidad Manuela Beltrán (Bucaramanga, Colombia). Dirección: Cl. 33 n. 27-12, PBX (60) (7) 6525202. ORCID ID: https://orcid.org/0000-00018157-6578. Correo electrónico: soniaastrid@gmail.com.
} 
Cindy Tatiana Daza Ríos, Sonia Astrid Reyes Duarte

Comportamientos ambientales en el sector turismo: revisión de tema

complete strategies focused on behavior change. Lastly, it is concluded that it is necessary to use a comprehensive approach in the strategies that seek to promote environmental behaviors in the tourism sector.

Keywords: Behavior, environmental conservation, ecology, environment, tourism.

\section{Introducción}

En la actualidad se necesitan consumidores y empresas que sean más conscientes de los impactos ambientales de sus actividades. El sector turismo ha sido importante en la contribución al crecimiento económico a nivel nacional e internacional, pero desde décadas pasadas existen preocupaciones sobre los efectos de esta actividad para el medio ambiente, particularmente en los países donde esta se constituye en una importante fuente de ingreso (Getz, 1983; McCool y Lime, 2001). Se suma a este deterioro ambiental, los efectos colaterales que trae el turismo, como el deterioro del patrimonio natural, cultural y la generación de problemas sociales como prostitución y tráfico de drogas, entre otros (Hernández, 2017). En este sentido, si se quiere contribuir al desarrollo sostenible, se requiere la generación de conocimiento que promueva el turismo sostenible. Por eso la necesidad de realizar una revisión del tema con el objetivo de conocer los últimos estudios desarrollados sobre este asunto y poder utilizar esta información para plantear posibles soluciones.

La Organización Mundial del Turismo (OMT) es un organismo que pertenece a la Organización de las Naciones Unidas (ONU) y es la encargada a nivel internacional de promover el turismo. La OMS define el turismo como "un fenómeno social, cultural y económico que supone el desplazamiento de personas a países o lugares fuera de su entorno habitual por motivos personales, profesionales o de negocios" (Organización Mundial del Turismo[OMT], 2008).

En Colombia, quien fomenta el turismo es el Ministerio de Comercio, Industria y Turismo, el cual ha emitido una normatividad que busca regular el sector. Con base en lo anterior, es especialmente importante la Ley 300 de 1996 (Congreso de la República, 1996), por la cual se expide la ley general de turismo modificada por la Ley 1558 de 2012 (Congreso de la República, 2012), que tiene por objeto regular la actividad turística que resguarda el desarrollo sostenible. Considerando la riqueza de Santander en biodiversidad, el departamento le ha apostado a este sector como un eje estratégico de la economía. Como muestra Hernández (2017) en sus resultados de investigación en los últimos años el Gobierno ha invertido en infraestructura con el objetivo de aumentar la visita de turistas, además de contar con la riqueza en paisajes naturales en el departamento.

A nivel internacional y nacional se promueve el turismo y la necesidad de fomentar e implementar estrategias de sostenibilidad en este sector. La mayoría de investigaciones sobre estos aspectos se centran únicamente en factores humanos o personales sin considerar factores higiénicos o de infraestructura. En este punto es preciso citar la investigación realizada por Wang, et al. (2020), quienes estudiaron la relación del turista con el medio ambiente $y$, en la cual, se realizan diferentes comportamientos ambientales que ponen en consideración las facilidades higiénicas. Esta visión integral de la elección del comportamiento incluyendo factores personales y factores situacionales es necesaria para tratar de diseñar estrategias que realmente contribuyan a generar comportamientos proambientales.

La pregunta que busca responder esta investigación con base en la revisión de información de fuentes especializadas es: ¿Qué información se ha publicado sobre comportamientos ambientales y factores higiénicos en el sector turismo? Para poder plantear investigaciones que puedan aportar posibles soluciones gracias a la información publicada hasta la fecha.

\section{Comportamientos ambientales en el sector turismo}

Los estudios sobre la relación entre las actitudes y los comportamientos ambientales fueron muy investigados en los años ochenta y noventa (Eilam y Trop, 2012). Estos estudios daban más importancia a la creación de comportamientos ambientales que a la creación de actitudes ambientales; Eilam y Trop (2012) contradijeron esto al sugerir un enfoque más crucial sobre las actitudes que sobre los comportamientos, contrario a los modelos tempranos lineales donde se considera al comportamiento como resultado de las actitudes. A pesar de que estos modelos fueron desacreditados, la idea del comportamiento ambiental como último objetivo se ha mantenido, aunque investigadores hayan concluido que las actitudes pueden ser irrelevantes para predecir los comportamientos. Otras teorías diferentes a los modelos tradicionales plantean que, ante un cambio de comportamiento, el cambio de actitud servirá para adaptarse al nuevo comportamiento e igualmente se ha reportado que la estabilidad de la actitud es mayor en personas mayores que en jóvenes.

Una investigación realizada por Amérigo et al. (2017) muestra cómo en la literatura se han realizado estudios que enfrentan las dimensiones de ecocentrismo y antropocentrismo. Sin embargo, una investigación realizada con jóvenes rechaza esta visión dual especialmente en Latinoamérica. Según Karpudewan et al. (2012), los 
países industrializados están aumentando la consciencia sobre el cuidado del medio ambiente, pero en los países en vía de desarrollo la situación es diferente.

Kovács et al. (2014) mencionaron en sus investigaciones que la mayoría de estudios muestran una asociación positiva entre actitudes y comportamientos ambientales, que es coherente con los modelos más citados para explicar esta relación como son la teoría de comportamiento planificado, Modelo de activación de normas y Modelo de valor, creencia, norma. Como complemento a lo anterior, los investigadores Park et al. (2018) en su trabajo mencionaron que se debe revisar una integración de los diferentes modelos que tratan de predecir el comportamiento humano con el objetivo de mejorar la predicción. Se encontró que la percepción del riesgo de los individuos influye positivamente en sus actitudes y comportamientos ambientales, por consiguiente, es importante emplear en las investigaciones la variable asociada al riesgo ocasionada por el cambio climático (Fang y Yu, 2015). Como lo mencionan Wu y Lu (2013) en un estudio realizado en la ciudad de Shenzhen en China, también es importante identificar los problemas ambientales porque el comportamiento ambiental responde directamente a estos.

Según Zheng et al. (2018), las crisis han facilitado a los seres humanos entender que los recursos naturales no son inagotables, además plantean como solución la promoción de conocimientos y actitudes ambientales. Los investigadores Arı y Yılmaz (2017) reportan cómo en varios estudios han encontrado mayor interés por comportamientos proambientales en mujeres que en hombres. Otros investigadores como Cheung et al. (2019) han estudiado la variable de orientación política encontrando niveles más altos de cultura y comportamiento ambiental en liberales que en conservadores, quienes son los menos preocupados por el ambiente en Hong Kong.

Una investigación realizada por Gao (2018) muestra que es importante generar conciencia para cambiar la forma de ver el mundo. En busca de contribuir con la generación de conocimiento sobre estos temas en México, Vanegas et al. (2018) realizaron una investigación que intentó establecer la relación entre la expectativa del comportamiento ambiental de otros y el autorreporte, lo que confirma la relación existente. Así mismo, en otros países como Brasil y Portugal se han desarrollado investigaciones que comparan el comportamiento ambiental en diferentes contextos y se han encontrado diferencias (Côrtes et al., 2016). Por su parte, CuervoArango et al. (2013) indican que comportarse de forma proambiental, además de favorecer el medio ambiente, se relaciona con un mayor nivel de bienestar emocional.

Diferentes investigaciones mencionan que es muy importante generar competencias que promuevan el comportamiento proambiental. Sobre este asunto
Rivera-Torres y Garcés-Ayerbe (2018) mencionan en su investigación conceptos de diferentes autores sobre comportamiento ambiental. Una de estas define el comportamiento: "como aquel que es adoptado con la intención de cambiar el medio ambiente" (p. 60) así mismo se refieren al comportamiento proambiental como "el comportamiento para no perjudicar al medio ambiente" (p.60).

\section{Metodología}

\section{Tipo de Estudio}

La investigación desarrollada tuvo un enfoque cualitativo y un alcance descriptivo. El artículo se desarrolló por medio de una revisión bibliográfica del tema en fuentes especializadas y para ello se utilizó una ecuación de búsqueda en las bases de datos Scielo y Scopus, la cual se presenta en la Tabla 1.

Tabla 1

Ecuación de búsqueda utilizada

\begin{tabular}{cc}
\hline Scopus & $\begin{array}{c}\text { (tourism AND ("environmental } \\
\text { behaviors" OR "environmental } \\
\text { attitudes" OR "hygienic factors")) } \\
\text { (turismo AND ("comportamientos } \\
\text { Scielo }\end{array} \begin{array}{c}\text { ambientales" OR "actitudes ambientales" } \\
\text { OR "factores higiénicos")) }\end{array}$ \\
\hline
\end{tabular}

Fuente: Autores

\section{Procedimiento}

Se realizó la búsqueda en la base de datos Scielo sin obtener resultados; mientras que en Scopus se obtuvo que en los últimos años ha aumentado la literatura científica publicada sobre el tema de estudio. Esto se ve reflejado en el aumento de artículos publicados como se observa en la Tabla 2

Tabla 2

Artículos sobre comportamientos ambientales en el sector turismo en los últimos cuatro año

\begin{tabular}{ccc}
\hline Año & Artículos publicados & Base de Datos \\
\hline 2019 & 41 & \\
2018 & 36 & Scopus \\
2017 & 16 & \\
2016 & 14 & \\
\hline
\end{tabular}

Fuente: Autores

Por su parte, los países con mayor número de investigaciones publicadas son Estados unidos, seguido de Reino Unido y en tercer lugar China.

Posteriormente, se delimitó la búsqueda al aplicarse el criterio de idiomas español e inglés. Los resultados también se restringieron a las publicaciones desde el 
Cindy Tatiana Daza Ríos, Sonia Astrid Reyes Duarte

Comportamientos ambientales en el sector turismo: revisión de tema
2016 en adelante, considerando los últimos 5 años y, de esta forma, se obtuvieron 124 documentos. Luego se filtró por área de conocimiento y se delimitó al área de Business, Management and Accounting. Se consiguieron 87 resultados y posteriormente se aplicó el criterio de tipo de documento, en el que solo se consideraron artículos en estado de publicación final y con información procedente de revista como fuente; esta acción redujo la búsqueda a 51 documentos.

Por último, se seleccionaron aquellos documentos que especificaron la recolección de datos de fuentes primarias y no se incluyeron revisiones documentales, al finalizar, el resultado fue 30 artículos.

\section{Resultados}

Dentro de los resultados más importantes de la revisión del tema se encuentra lo reportado por Liu et al. (2020), quienes indican que los hábitos son predictores poderosos del comportamiento; los autores investigaron cómo los comportamientos ecológicos diarios de los visitantes influían en su comportamiento ambiental en destinos turísticos. Por ello, es importante gestionar los comportamientos de los turistas. Además, un estudio del 2018 estimó que la huella del carbono del turismo representa casi el $10 \%$ de las emisiones de gases de efecto invernadero y otros investigadores han reportado que el turismo es peligroso para el medio ambiente. Sin embargo, debido a la importancia de esta actividad para el crecimiento económico y el desarrollo social, es necesario realizar una gestión ambiental adecuada para poder ejecutar el turismo previniendo o minimizando los impactos ambientales negativos.

Esta investigación estudió los comportamientos ecológicos diarios según la teoría del comportamiento planificado asociados a los comportamientos proambientales, pero no solo como otros estudios lo han investigado, es decir, como antecedente, sino también como moderador encontrando resultados positivos que refuerzan este hallazgo, quizás porque si un individuo realiza comportamientos proambientales habituales es más probable que simplifique su proceso de decisión y se comporte de acuerdo a este. Los investigadores encontraron que promover hábitos de vida sostenible diarios sirve para promover el turismo sostenible.

Por su parte, Wang, Wang, Li y Zhou (2020) estudiaron el efecto de la religiosidad en el comportamiento proambiental de turistas en hoteles; la religiosidad intrínseca afectó positivamente, no siendo el caso de la religiosidad extrínseca. Estos estudios en los hoteles son muy importantes debido a que, por un lado, tienen un impacto positivo en el ambiente y por otro se ha generado un aumento del enfoque en el turismo verde y sostenible en los últimos años, pero estas prácticas necesitan además de la participación de los hoteles y los turistas. Peng y Lee (2019) también estudiaron las motivaciones de los trabajadores de hoteles para poner en práctica comportamientos proambientales y mencionan la importancia de la ética laboral y la evaluación de externalidades como la salud en el ambiente de trabajo, la comunidad y los beneficios económicos para las empresas; por consiguiente, es importante promover en las organizaciones la responsabilidad ambiental como estrategia competitiva.

Por su parte, Zhang y Huang (2019) realizaron estudios con trabajadores de hoteles para promover comportamientos ambientales adecuados. Las empresas deben considerar e implementar esto, no solo por el ahorro asociado a la eficiencia, sino también por la imagen positiva de la marca (Okumus et al., 2019). Las investigaciones muestran cómo los gerentes de los destinos de turismo tienen varios aspectos por mejorar, por ejemplo, el transporte lento debido a la disminución de gases de efecto invernadero que se asocia a estas prácticas y a la mejora en la experiencia por parte del turista. Según lo reportando por Lin (2018), se deben promover los beneficios de este tipo de transporte para los turistas y diseñar estrategias específicas que faciliten estos comportamientos.

Los investigadores Han y Hyun (2018) estudiaron comportamientos de compra ecológica y reciclaje de los viajeros, estas también son prácticas muy importantes que se deben considerar y buscar promover en las estrategias diseñadas sobre turismo sostenible. De acuerdo con Zhang et al. (2018), los gerentes de los sitios turísticos y mandatarios locales podrían realizar campañas con líderes sobre comportamientos ambientales, pues ellos, en su estudio sobre comportamientos de turistas en parques urbanos, mencionaron la importancia de la percepción de la presión social en sus decisiones más que otros aspectos. Los investigadores Landon et al. (2018) mencionan en su estudio que los turistas y las comunidades son fundamentales para la sostenibilidad de este sector $y$, por lo tanto, todas las variables reportadas deben ser consideradas y puestas en práctica lo antes posible.

Como lo describen Wang, Wang, Li y Zhou (2020), los residentes locales son importantes en la generación de comportamientos proambientales $y$, actualmente, se estudia el cómo disminuir los impactos ambientales negativos del turismo y las estrategias que se planteen deben considerar las diferentes partes interesadas. Este estudio buscó estudiar los motivadores del comportamiento de los residentes y se encontró que la obligación sentida y la preocupación altruista afectan directa y positivamente los comportamientos proambientales de los residentes. También las empresas deben aprovechar los comentarios de los turistas sobre el desempeño verde de las organizaciones y trabajar de forma conjunta para co-crear valor (Luu, 2018).

Por otra parte, los investigadores Lin y Lee (2020) estudiaron cómo afectan las experiencias recreativas a los 
Cindy Tatiana Daza Ríos, Sonia Astrid Reyes Duarte Comportamientos ambientales en el sector turismo: revisión de tema

visitantes de senderos antiguos en Taiwán encontrando que la experiencia recreativa está relacionada de forma directa con las actitudes ambientales y el apego al lugar e indirectamente relacionada con los comportamientos ambientales.

Zhang et al. (2020) estudiaron la relación entre el desarrollo social y el cuidado ambiental. Esta investigación fue desarrollada en China y sugiere que la percepción de los cambios en los capitales de medios de vida tiene un impacto significativo en el comportamiento proambiental de los residentes. La sugerencia de envolver a la comunidad para generar comportamientos ambientalmente responsables de los residentes también es respaldada por Safshekan et al. (2020), quienes mencionan la importancia de considerar a los residentes en la formulación de estrategias que promuevan el turismo sostenible.

Li y Wu (2019) han estudiado los modelos de comportamiento que explican mejor el comportamiento de turistas locales y no locales, encontraron diferencias en la explicación de los comportamientos de las dos poblaciones: en los visitantes funcionan los dos modelos del comportamiento planeado y la teoría de activación de la norma, mientras que, en los visitantes no locales, influye predominantemente la moralidad. Estas investigadoras concluyen con la importancia de generar un modelo integrador que tenga una perspectiva holística y en la cual se consideren las percepciones de los residentes, porque a pesar de los beneficios resultado de los impactos positivos, también pueden presentarse preocupaciones sobre los impactos negativos tal como lo menciona Md Noor et al. (2019) en su investigación.

Por su parte, Mokhtari et al. (2019) resaltaron en su investigación sobre geoturismo la importancia de los lugares protegidos en el mantenimiento de la biodiversidad y los sistemas naturales. Los investigadores concluyen que es necesario considerar las facilidades de infraestructura que generen la oportunidad de realizar comportamientos adecuados como mapas de la zona y facilidades higiénicas como recipientes para arrojar residuos. Como lo indican D'Souza et al. (2019), se debe buscar generar una relación de simbiosis entre turistas y la comunidad residente. Los beneficios no son solo económicos, también aporta a la salud en general, debido a una adecuada calidad del medio ambiente.

En tanto, los investigadores Hanna et al. (2019) reportan que las actividades de turismo de aventura al aire libre tienen implicaciones en la salud mental y bienestar. También el turismo silvestre como la visita a acuarios, zoológicos y avistamiento de ballenas ofrece la oportunidad de promover el aumento de los valores, actitudes y comportamientos proambientales como lo mencionaron Clark et al. (2019) y Ballantyne et al. (2018). Según Wicker (2018), los turistas que realizan deportes pareciera que valoran las prácticas ecológicas. Las anteriores investigaciones muestran cómo diferentes tipos de turismo pueden utilizarse como herramientas para promover el turismo sostenible.

Las investigaciones que aportan a la sostenibilidad deben ser interdisciplinarias, como lo abordaron en su investigación Tussyadiah y Miller (2019) la inteligencia artificial y en general la tecnología podría ser útil para promover un cambio positivo en los comportamientos. Otras variables importantes que se deben estudiar y no han sido muy consideradas son el pesimismo espacial y el optimismo temporal (Zhang et al., 2019). También los mecanismos de comunicación utilizados para promover los comportamientos proambientales en los turistas son muy importantes, como lo encontraron en su investigación Gössling et al. (2019) y Grazzini et al. (2018); por ejemplo, las redes sociales como Facebook podrían utilizarse para promover comportamientos amigables con el medio ambiente (Han et al., 2018). Por consiguiente, se puede ver cómo todavía falta generar conocimiento sobre todo para romper la "brecha entre actitud e intención" como lo mencionaron en su investigación $\mathrm{Qu}$ et al. (2019) y finalmente el cambio de comportamiento.

\section{Discusión}

Según la investigación realizada por Liu et al. (2020) se requieren más estudios para esclarecer la relación entre actitud y comportamientos ambientales. De acuerdo a la teoría del comportamiento planificado, cuando se cambia una actitud se promueve un cambio de comportamiento y cuando la actitud es apoyada por normas subjetivas y control percibido conduce a la intención de comportamiento y acciones, sin embargo, otros investigadores han reportado inconsistencias sobre esto (Eilam y Trop, 2012).

Safshekan et al. (2020) mencionaron en sus investigaciones que las herramientas que buscan promover el turismo sostenible no deben considerar solo a los turistas, también se deben considerar otras partes interesadas de los destinos turísticos. Este punto de vista es compartido por Vollero et al. (2018) quienes consideran que los residentes son importantes. Además, las estrategias se deben estructurar formalmente y considerar políticas y procedimientos (Wang, Wang, Li, y Yang, 2020) y las facilidades higiénicas (Wang, Zhang et al., 2020) para buscar promover comportamientos sostenibles considerando los diferentes aspectos que influyen en la decisión del comportamiento, según las teorías más importantes que buscan explicar las causas y predecir los comportamientos. Los investigadores D'Souza et al. (2019) encontraron que las organizaciones deben buscar, además de motivar incentivos para promover estos comportamientos amigables con el medio ambiente, promover comportamientos asociados a la ética del trabajo y dar a conocer a sus colaboradores los beneficios de implementar estas prácticas, coincidiendo con Hanna 
Cindy Tatiana Daza Ríos, Sonia Astrid Reyes Duarte

Comportamientos ambientales en el sector turismo: revisión de tema

et al. (2019) y Okumus et al. (2019), quienes establecieron la importancia de comunicar los beneficios a diferentes partes interesadas.

\section{Conclusiones}

Para promover comportamientos y actitudes amigables con el medio ambiente se requiere de evidencia científica válida que aclare cómo es la relación entre estas dos variables con el objetivo de poder diseñar estrategias pertinentes, considerando hábitos ecológicos diarios, porque como la literatura lo reportó, estos funcionan como antecedente y moderador del comportamiento. Estos hábitos en la vida diaria son vitales para el desarrollo y el turismo sostenible siendo necesaria la capacitación de los empleados de este sector y la promoción de un clima ambiental adecuado por parte de las organizaciones, los residentes y en general todos los ciudadanos y potenciales turistas se deben considerar en la formulación de estrategias que promuevan el turismo sostenible.

La revisión bibliográfica muestra cómo el comportamiento ambiental debe estudiarse desde una visión holística e integrando diferentesmodelos tradicionales deexplicación del comportamiento porque al integrarlos, aumenta la probabilidad de predicción del comportamiento. Se requiere contemplar además de factores personales, factores higiénicos o situaciones que influyen en la decisión final del comportamiento a ejecutar. Se considera importante integrar diferentes paradigmas cognitivos en el diseño de estrategias y, sobre todo, profundizar en los problemas ambientales existentes y la percepción del riesgo asociada al cambio climático.

Se deben desarrollar investigaciones en el contexto colombiano con el objetivo de generar conocimiento sobre cómo se comportan las personas y aportar información para tratar de entender cómo es el proceso de toma de decisión de comportamiento ambiental en este contexto.

Por último, se puede concluir que la investigación en estos temas ha aumentado en los últimos años al considerar el incremento en las publicaciones; sumado a lo anterior, el idioma predominante en el cual se divulga el conocimiento es el inglés y que los países con mayor número de publicaciones son potencias como Estados Unidos, Reino Unido y China.

\section{Agradecimientos}

Agradecimiento especial al Dr. Reynaldo Rodríguez por los conocimientos aportados.

\section{Referencias}

Amérigo, M., García, J. A., y Côrtes, P. L. (2017). Analysis of environmental attitudes and behaviors: an exploratory study with a sample of brazilian university students. Ambiente \& Sociedade, 20(3), 1-20. https://doi.org/10.1590/1809-4422asoc300r1 v2032017

Arı, E., y Yılmaz, V. (2017). Effects of environmental illiteracy and environmental awareness among middle school students on environmental behavior. Environment, Development and Sustainability, 19(5), 1779-1793. https://doi.org/10.1007/s10668-016-982 6-3

Ballantyne, R., Hughes, K., Lee, J., Packer, J., y Sneddon, J. (2018). Visitors' values and environmental learning outcomesatwildlifeattractions:Implicationsforinterpretive practice. Tourism Management, 64, 190-201. https://doi.org/10.1016/j.tourman.2017.07.01 5

Cheung, L. T. O., Ma, A. T. H., Lee, K. M. Y., Lee, J. C. K., y Lo, Y. L. (2019). How does political orientation influence one's environmental attitude and behaviour? Debate over country park conservation in Hong Kong. Environmental Science \& Policy, 99, 115-122. https://doi.org/10.1016/j.envsci.2019.05.026

Clark, E., Mulgrew, K., Kannis-Dymand, L., Schaffer, V., y Hoberg, R. (2019). Theory of planned behaviour: predicting tourists' pro-environmental intentions after a humpback whale encounter. Journal of Sustainable Tourism, 27(5), 649-667. https://doi. org/10.1080/09669582.2019.1603237

Congreso de la República. (1996). Ley 300 de 1996. https:// www.funcionpublica.gov.co/eva/gestornormativo/ norma.php?i=8634

Congreso de la República. (2012). Ley 1558 de 2012. http:// www.secretariasenado.gov.co/senado/basedoc/ ley_1558_2012.html

Côrtes, P. L., Guerner Dias, A., Da Silva Teixeira, M. E., y Vieira Pamplona, J. M. (2016). Environmental behavior: a comparative study between brazilian and portuguese students. Ambiente y Sociedade, 19(3), 113-134. https://doi.org/10.1590/1809-4422A SOC139099V1932016

Cuervo-Arango, A. M., García, J. A., y Sánchez Nuñez, T. (2013). Actitudes y comportamiento hacia el medio ambiente natural. Salud medioambiental y bienestar emocional. Universitas Psychologica, 12(3), 845-856. https://doi.org/10.11144/Javeriana. UPSY12-3.acma 
Cindy Tatiana Daza Ríos, Sonia Astrid Reyes Duarte Comportamientos ambientales en el sector turismo: revisión de tema

D'Souza, C., Taghian, M., Marjoribanks, T., SullivanMort, G., Manirujjaman, M. D., y Singaraju, S. (2019). Sustainability for ecotourism: work identity and role of community capacity building. Tourism Recreation Research, 44(4), 533-549. https://doi.or $\mathrm{g} / 10.1080 / 02508281.2019 .1654727$

Eilam, E., y Trop, T. (2012). Environmental attitudes and environmental behavior-which is the horse and which is the cart? Sustainability, 4(9), 2210-2246. https://doi.org/10.3390/su4092210

Fang, S.-C., y Yu, T.-Y. (2015). A Risk Perception Model Of Climate Change For University Students. Journal of Baltic Science Education, 14(3), 339-350.

Gao, Y. (2018). To Study the Relationship between Environmental Education and Environmental Behavior Based on Environmental Attitude. Ekoloji, 27(106), 627-634.

Getz, D. (1983). Capacity to absorb tourism: Concepts and implications for strategic planning. Annals of Tourism Research, 10(2), 239-263. https://doi. org/10.1016/0160-7383(83)90028-2

Gössling, S., Araña, J. E., y Aguiar-Quintana, J. T. (2019). Towel reuse in hotels: Importance of normative appeal designs. Tourism Management, 70, 273-283. https://doi.org/10.1016/j.tourman.2018.08.027

Grazzini, L., Rodrigo, P., Aiello, G., y Viglia, G. (2018). Loss or gain? The role of message framing in hotel guests' recycling behaviour. Journal of Sustainable Tourism, 26(11), 1944-1966. https://doi.org/10.1080/0966958 2.2018.1526294

Han, H., y Hyun, S. (2018). College youth travelers' eco-purchase behavior and recycling activity while traveling: an examination of gender difference. Journal of Travel \& Tourism Marketing, 35(6), 740-754. https://doi.org/10.1080/10548408.2017.1405865

Han, W., McCabe, S., Wang, Y., y Chong, A. Y. L. (2018). Evaluating user-generated content in social media: an effective approach to encourage greater proenvironmental behavior in tourism? Journal of Sustainable Tourism, 26(4), 600-614. https://doi. org/10.1080/09669582.2017.1372442

Hanna, P., Wijesinghe, S., Paliatsos, I., Walker, C., Adams, M., y Kimbu, A. (2019). Active engagement with nature: outdoor adventure tourism, sustainability and wellbeing. Journal of Sustainable Tourism, 27(9), 1355-1373. https://doi.org/10.1080/09669582.2019. 1621883

Hernández, Y. (2017). Caracterización del sector turismo empresarios de Bucaramanga y su área metropolitana.
Universidad Santo Tomas. https://rep os itory.usta. edu.co/handle/11634/4775

Karpudewan, M., Ismail, Z., y Roth, W.-M. (2012). Promoting pro-environmental attitudes and reported behaviors of Malaysian pre-service teachers using green chemistry experiments. Environmental Education Research, 18(3), 375-389. https://doi.org/10.1080/13504622.2011.622841

Kovács, J., Pántya, J., Medvés, D., Hidegkuti, I., Heim, O., y Bursavich, J. B. (2014). Justifying environmentally significant behavior choices: An AmericanHungarian cross-cultural comparison. Journal of Environmental Psychology, 37, 31-39. https://doi. org/10.1016/j.jenvp.2013.11.001

Landon, A. C., Woosnam, K. M., y Boley, B. B. (2018). Modeling the psychological antecedents to tourists' pro-sustainable behaviors: an application of the value-belief-norm model. Journal of Sustainable Tourism, 26(6), 957-972. https://doi. org/10.1080/09669582.2017.1423320

Li, Q. C., y Wu, M. Y. (2019). Rationality or morality? A comparative study of pro-environmental intentions of local and nonlocal visitors in nature-based destinations. Journal of Destination Marketing and Management, 11(Julio 2018), 130-139. https://doi. org/10.1016/j.jdmm.2019.01.003

Lin, L. (2018). How would the contextual features of a destination function together with individual factors to enhance tourists' intention toward ST in Taiwan? Journal of Sustainable Tourism, 26(9), 1625-1646. https://doi.org/10.1080/09669582.2018 .1491586

Lin, Y. H., y Lee, T. H. (2020). How do recreation experiences affect visitors' environmentally responsible behavior? Evidence from recreationists visiting ancient trails in Taiwan. Journal of Sustainable Tourism, 28(5), 705-726. https://doi.org/10.1080/09 669582.2019 .1701679

Liu, A., Ma, E., Qu, H., y Ryan, B. (2020). Daily green behavior as an antecedent and a moderator for visitors' pro-environmental behaviors. Journal of Sustainable Tourism, 28(9), 1390-1408. https://doi. org/10.1080/09669582.2020.1741598

Luu, T. T. (2018). Employees' green recovery performance: the roles of green HR practices and serving culture. Journal of Sustainable Tourism, 26(8), 1308-1324. https://doi.org/10.1080/09669582.2018.1443113

McCool, S. F. y Lime, D. W. (2001). Tourism Carrying Capacity: Tempting Fantasy or Useful Reality? 
Cindy Tatiana Daza Ríos, Sonia Astrid Reyes Duarte

Comportamientos ambientales en el sector turismo: revisión de tema

Journal of Sustainable Tourism, 9(5), 372-388. https:// doi.org/10.1080/09669580108667409

Md Noor, S., Rasoolimanesh, S. M., Jaafar, M., y Barghi, R. (2019). Inscription of a destination as a world heritage site and residents' perceptions. Asia Pacific Journal of Tourism Research, 24(1), 14-30. https://doi. org/10.1080/10941665.2018.1541183

Mokhtari, D., Roostaei, S., Khodadadi, M., Ahmadi, M., Ebrahimi, O., y Shahabi, H. (2019). Evaluation of the Role of Environmental Education in Manesht and Ghelarang Geotourism Destination, Iran. Journal of Quality Assurance in Hospitality \& Tourism, 20(6), 681-708. https://doi.org/10.1080/1528008X.2019.16 16039

Okumus, F., Köseoglu, M. A., Chan, E., Hon, A., y Avci, U. (2019). How do hotel employees' environmental attitudes and intentions to implement green practices relate to their ecological behavior? Journal of Hospitality and Tourism Management, 39, 193-200. https://doi.org/10.1016/j.jhtm.2019.04.008

Organización Mundial del Turísmo. (2008). Glosario de términos de turismo. UNWTO.Org. https://www. unwto.org/es/glosario-terminos-turisticos

Park, E., Lee, S. J., Lee, C. K., Kim, J. S., y Kim, N. J. (2018). An integrated model of travelers' pro-environmental decision-making process: the role of the New Environmental Paradigm. Asia Pacific Journal of Tourism Research, 23(10), 935-948. https://doi.org/1 $0.1080 / 10941665.2018 .1513051$

Peng, X., y Lee, S. (2019). Self-discipline or self-interest? The antecedents of hotel employees' pro-environmental behaviours. Journal of Sustainable Tourism, 27(9), 1457-1476. https://doi.org/10.1080/0 9669582.2019 .1632320

Qu, Y., Xu, F., y Lyu, X. (2019). Motivational place attachment dimensions and the pro-environmental behaviour intention of mass tourists: a moderated mediation model. Current Issues in Tourism, 22(2), 197-217. https://doi.org/10.1080/13683500.2017.1399988

Rivera-Torres, P., y Garcés-Ayerbe, C. (2018). Desarrollo del comportamiento proambiental en los individuos y sus determinantes / Development of ProEnvironmental Conduct in Individuals and its Determinants. Revista Española de Investigaciones Sociológicas, 163, 59-78. https://doi.org/10.5477/cis /reis.163.59

Safshekan, S., Ozturen, A., y Ghaedi, A. (2020). Residents' environmentally responsible behavior: an insight into sustainable destination development. Asia
Pacific Journal of Tourism Research, 25(4), 409-423. https://doi.org/10.1080/10941665.2020.1737159

Tussyadiah, I., y Miller, G. (2019). Nudged by a robot: Responses to agency and feedback. Annals of Tourism Research, 78. https://doi.org/10.1016/j.anna Is.2019.102752

Vanegas Rico, M. C., Corral-Verdugo, V., Ortega-Andeane, P., y Bustos-Aguayo, J. M. (2018). Desarrollo de la Escala Expectativa de Comportamiento Ambiental de Otros con adultos jóvenes mexicanos. Universitas Psychologica, 17(2), 1-10.

Vollero, A., Conte, F., Bottoni, G., y Siano, A. (2018). The influence of community factors on the engagement of residents in place promotion: Empirical evidence from an Italian heritage site. International Journal of Tourism Research, 20(1), 88-99. https://doi.org/10 $.1002 / j t r .2164$

Wang, C., Zhang, J., Sun, J., Chen, M., y Yang, J. (2020). Public environmental facilities: Hygiene factors for tourists' environmental behaviour. Environmental Science and Policy, 106, 40-47. https://doi.org/ 10.1016/j.envsci.2020.01.009

Wang, S., Wang, J., Li, J., y Yang, F. (2020). Do motivations contribute to local residents' engagement in pro-environmental behaviors? Resident-des tination relationship and pro-environmental climate perspective. Journal of Sustainable Tourism, 28(6), 834-852. https://doi.org/10.1080/09669582.2019.17 07215

Wang, S., Wang, J., Li, J., y Zhou, K. (2020). How and when does religiosity contribute to tourists' intention to behave pro-environmentally in hotels? Journal of Sustainable Tourism, 28(8), 1120-1137. https://doi. org/10.1080/09669582.2020.1724122

Wicker, P. (2018). The carbon footprint of active sport tourists: an empirical analysis of skiers and boarders. Journal of Sport \& Tourism, 22(2), 151-171. https://doi.org/10.1080/14775085.2017.1313706

Wu, X., y Lu, W. (2013). A Study on Environmental Attitude, Environmental Behavior and Environment Issues of Living Place: The Questionnaire Survey for some Dwellers in Shenzhen. Applied Mechanics and Materials, 368-370, 290-296. https://doi. org/10.4028/www.scientific.net/AMM.368-370.290

Zhang, H., Zhang, Y., Song, Z., y Lew, A. A. (2019). Assessment bias of environmental quality (AEQ), consideration of future consequences (CFC), and environmentally responsible behavior (ERB) in tourism. Journal of Sustainable Tourism, 27(5), 
609-628. https://doi.org/10.1080/09669582.2019.1

597102

Zhang,J.,yHuang,R.(2019).Employees'pro-environmental behaviours (PEBs) at international hotel chains (IHCS) in China:The mediating role of environmental concerns (ECs). Journal of Hospitality and Tourism Management, 39, 129-136. https://doi.org/10.1016/j. jhtm.2019.03.007

Zhang, Y., Moyle, B. D., y Jin, X. (2018). Fostering visitors' pro-environmental behaviour in an urban park. Asia Pacific Journal of Tourism Research, 23(7), 691-702. https://doi.org/10.1080/10941665.2018.1487457

Zhang, Y., Xiao, X., Zheng, C., Xue, L., Guo, Y., y Wu, Q. (2020). Is tourism participation in protected areas the best livelihood strategy from the perspective of community development and environmental protection? Journal of Sustainable Tourism, 28(4), 587-605. https://doi.org/10.1080/09669582.2019.16 91566

Zheng, W.-L., Wang, J.-W., y Zhang, X. (2018). Effects of Environmental Cognition and Environmental Attitude on Environmental Behavior of Ecotourism. Ekoloji, 27(106), 1743-1749. 